\title{
LIPSCHITZ CONTINUITY OF BLOCH TYPE MAPPINGS WITH RESPECT TO BERGMAN METRIC
}

\author{
Shaolin Chen and David Kalaj \\ Hengyang Normal University, College of Mathematics and Statistics \\ Hengyang, Hunan 421008, P. R. China; mathechen@126.com \\ and Soochow University, Suzhou, Jiangsu 215006, P. R. China \\ University of Montenegro, Faculty of Natural Sciences and Mathematics \\ Cetinjski put b. b. 81000 Podgorica, Montenegro; davidk@t-com.me
}

\begin{abstract}
In this paper, we establish the sharp estimate of the Lipschitz continuity with respect to the Bergman metric. The obtained results are the improvement and generalization of the corresponding results of Ghatage et al. [3] and Hosokawa et al. [4].
\end{abstract}

\section{Introduction and main results}

Let $\mathbf{C}^{n}$ denote the Euclidean space of complex dimension $n$. For $z=\left(z_{1}, \ldots, z_{n}\right) \in$ $\mathbf{C}^{n}$, the conjugate of $z$, denoted by $\bar{z}$, is defined by $\bar{z}=\left(\bar{z}_{1}, \ldots, \bar{z}_{n}\right)$. For $z$ and $w=\left(w_{1}, \ldots, w_{n}\right) \in \mathbf{C}^{n}$, we write

$$
\langle z, w\rangle:=z \cdot w=\sum_{k=1}^{n} z_{k} \bar{w}_{k} \quad \text { and } \quad|z|:=\langle z, z\rangle^{1 / 2}=\left(\sum_{k=1}^{n}\left|z_{k}\right|^{2}\right)^{1 / 2} .
$$

For $a=\left(a_{1}, \ldots, a_{n}\right) \in \mathbf{C}^{n}$, we set

$$
\mathbf{B}^{n}(a, r)=\left\{z \in \mathbf{C}^{n}:|z-a|<r\right\} .
$$

Also, we use $\mathbf{B}^{n}$ to denote the unit ball $\mathbf{B}^{n}(0,1)$ and let $\mathbf{D}=\mathbf{B}^{1}$. In the following, we always treat $z \in \mathbf{C}^{n}$ as a column vector, that is, $n \times 1$ column matrix

$$
z=\left(\begin{array}{c}
z_{1} \\
\vdots \\
z_{n}
\end{array}\right)
$$

The class of all holomorphic functions from $\mathbf{B}^{n}$ into $\mathbf{C}^{n}$ is denoted by $H\left(\mathbf{B}^{n}, \mathbf{C}^{n}\right)$. Let $\operatorname{Aut}\left(\mathbf{B}^{n}\right)$ be the automorphism group consisting of all biholomorphic self mappings of the unit ball $\mathbf{B}^{n}$ (cf. [8]).

For $z \in \mathbf{B}^{n}$, let

$$
B(z)=\frac{\left(1-|z|^{2}\right) I+A(z)}{\left(1-|z|^{2}\right)^{2}}
$$

be the Bergman matrix, where $I$ is the $n \times n$ identity matrix and

$$
A(z)=\left(\begin{array}{ccc}
z_{1} \bar{z}_{1} & \cdots & z_{1} \bar{z}_{n} \\
& & \\
\vdots & & \\
z_{n} \bar{z}_{1} & \cdots & z_{n} \bar{z}_{n}
\end{array}\right) .
$$

https://doi.org/10.5186/aasfm.2018.4309

2010 Mathematics Subject Classification: Primary 32A10; Secondary 32A18.

Key words: Holomorphic mapping, Lipschitz continuity, Bergman metric. 
For a smooth curve $\gamma:[0,1] \rightarrow \mathbf{B}^{n}$, let

$$
\ell(\gamma)=\int_{0}^{1}\left\langle B(\gamma(t)) \gamma^{\prime}(t), \gamma^{\prime}(t)\right\rangle^{1 / 2} d t
$$

For any two points $z$ and $w$ in $\mathbf{B}^{n}$, let $\beta(z, w)$ be the infimum of the set consisting of all $\ell(\gamma)$, where $\gamma$ is a piecewise smooth curve in $\mathbf{B}^{n}$ from $z$ to $w$. Then we call $\beta$ the Bergman metric in $\mathbf{B}^{n}$ (cf. [11]).

The prenorm $\|f\|_{\mathcal{P}(n, \alpha)}$ of $f \in H\left(\mathbf{B}^{n}, \mathbf{C}^{n}\right)$ is given by

$$
\|f\|_{\mathcal{P}(n, \alpha)}=\sup _{z \in \mathbf{B}^{n}} D_{f}^{n, \alpha}(z)
$$

where $\alpha>0$ and

$$
D_{f}^{n, \alpha}(z)=\left(1-|z|^{2}\right)^{\frac{\alpha(n+1)}{2 n}}\left|\operatorname{det} f^{\prime}(z)\right|^{\frac{1}{n}}
$$

For more details on prenorm, we refer to [6].

Let $\mathcal{B}_{\mathcal{P}(n, \alpha)}$ be the class of all holomorphic mappings $f \in H\left(\mathbf{B}^{n}, \mathbf{C}^{n}\right)$ satisfying $\|f\|_{\mathcal{P}(n, \alpha)}<\infty$. We call $f \in \mathcal{B}_{\mathcal{P}(n, \alpha)}$ a Bloch type mappings. In particular, $\mathcal{B}_{\mathcal{P}(1, \alpha)}$ is the classical family of $\alpha$-Bloch functions (cf. [10, 11]).

For $z, w \in \mathbf{D}$, the pseudo-hyperbolic distance is defined as

$$
\rho(z, w)=\left|\frac{z-w}{1-\bar{w} z}\right| .
$$

In particular, if $n=1$, then, for $z, w \in \mathbf{D}, \tanh \beta(z, w)=\rho(z, w)$. In [3], Ghatage et al. showed that $D_{f}^{1,1}(z)$ is Lipschitz continuous with respect to the pseudo-hyperbolic metric, which is given in Theorem A below.

Theorem A. [3, Theorem 1] Let $f \in \mathcal{B}_{\mathcal{P}(1,1)}$. Then, for all $z, w \in \mathbf{D}$,

$$
\left|D_{f}^{1,1}(z)-D_{f}^{1,1}(w)\right| \leq C\|f\|_{\mathcal{P}(1,1)} \rho(z, w),
$$

where $C=3.31$.

We remark that the constant $C=3.31$ in Theorem 1 is not sharp.

In [5], Hosokawa and Ohno proved

$$
\left|D_{f}^{1,1}(z)-D_{f}^{1,1}(w)\right| \leq 20\|f\|_{\mathcal{P}(1,1)} \rho(z, w),
$$

and they used it to discussed the composition operators on the Bloch spaces (cf. $[4,5])$. For more details on this topic, see $[1,2,7,9]$.

Let $\varphi$ be a holomorphic mapping of $\mathbf{B}^{n}$ into $\mathbf{B}^{n}$. For all $f \in \mathcal{B}_{\mathcal{P}(n, \alpha)}$, let $C_{\varphi}: f \mapsto$ $f \circ \varphi$ be a composition operator. As an application of Theorem A, Ghatage et al. [3] proved

Theorem B. [3, Theorem 2] Let $\varphi$ be a holomorphic mapping of $\mathbf{D}$ into $\mathbf{D}$. If for some constants $r \in(0,1 / 4)$, and $\varepsilon>0$, for each $w \in \mathbf{D}$, there is a point $z_{w} \in \mathbf{D}$ such that

$$
\rho\left(\varphi\left(z_{w}\right), w\right)<r, \quad \text { and } \quad \frac{1-\left|z_{w}\right|^{2}}{1-\left|\varphi\left(z_{w}\right)\right|^{2}}\left|\varphi^{\prime}\left(z_{w}\right)\right|>\varepsilon,
$$

then $C_{\varphi}: \mathcal{B}_{\mathcal{P}(1,1)} \rightarrow \mathcal{B}_{\mathcal{P}(1,1)}$ is bounded below.

In this paper, by using a different method, we generalize Theorems A and B to several dimensional case and obtain the sharp estimate of the Lipschitz constant with respect to the Bergman metric. 
Theorem 1. Let $f \in \mathcal{B}_{\mathcal{P}(n, 1)}$. Then, for $z_{1}, z_{2} \in \mathbf{B}^{n}$,

$$
\left|D_{f}^{n, 1}\left(z_{2}\right)-D_{f}^{n, 1}\left(z_{1}\right)\right| \leq M(n)\|f\|_{\mathcal{P}(n, 1)}\left[\tanh \beta\left(z_{1}, z_{2}\right)\right]^{\frac{1}{n}},
$$

where $M(n)=(2+n)^{\frac{1}{2 n}}\left(\frac{n+2}{n+1}\right)^{\frac{n+1}{2 n}}$. Moreover, the constant $M(n)$ in (2) cannot be replaced by a smaller number.

The following result is an application of Theorem 1 .

Theorem 2. Let $f \in \mathcal{B}_{\mathcal{P}(1,1)}$. Then, for $z \in \mathbf{D}$,

$$
\left(1-|z|^{2}\right)\left(\left|\frac{\partial}{\partial z} D_{f}^{1,1}(z)\right|+\left|\frac{\partial}{\partial \bar{z}} D_{f}^{1,1}(z)\right|\right) \leq \frac{3 \sqrt{3}}{2}\|f\|_{\mathcal{P}(1,1)}
$$

and

$$
\left|f^{\prime \prime}(z)\right| \leq \frac{\left(2|z|+\frac{3 \sqrt{3}}{2}\right)\|f\|_{\mathcal{P}(1,1)}}{\left(1-|z|^{2}\right)^{2}} .
$$

Moreover, the extreme functions $f(z)= \pm 3 \sqrt{3} z^{2} / 4$ show that the estimates (3) and (4) are sharp.

Applying Theorem 1, we get the following result which is an improvement of Theorem A.

Theorem 3. Let $\varphi$ be a holomorphic mapping of $\mathbf{B}^{n}$ into $\mathbf{B}^{n}$. Suppose that there is constants $0<r<\frac{1}{M(n)}\left(\frac{n+2}{1+n}\right)^{\frac{1}{n}}$ and $\varepsilon>0$ such that, for each $w \in \mathbf{B}^{n}$, there is a point $z_{w} \in \mathbf{B}^{n}$ satisfying $\tanh \beta\left(\varphi\left(z_{w}\right), w\right)<r^{n}$ and $\left|\tau_{\varphi}\left(z_{w}\right)\right|>\varepsilon$, where

$$
\tau_{\varphi}\left(z_{w}\right)=\left(\frac{1-\left|z_{w}\right|^{2}}{1-\left|\varphi\left(z_{w}\right)\right|^{2}}\right)^{\frac{n+1}{2 n}}\left|\operatorname{det} \varphi^{\prime}\left(z_{w}\right)\right|^{\frac{1}{n}}
$$

and $M(n)$ is defined as in Theorem 1. Then, for all $f \in \mathcal{B}_{\mathcal{P}(n, 1)}$, there is a constant $k(n, r, \varepsilon)>0$ depended only on $r, \varepsilon$ and $n$ such that

$$
\left\|C_{\varphi}(f)\right\|_{\mathcal{P}(n, 1)} \geq k(n, r, \varepsilon)\|f\|_{\mathcal{P}(n, 1)} .
$$

The proofs of Theorems 1-3 will be presented in Section 2.

\section{Proofs of the main results}

We begin the section by recalling the following results which play an important role in the proofs of Theorem 1.

Lemma C. [2, Lemma 1.1] For $x \in[0,1]$, let

$$
\varphi(x)=x\left(1-x^{2}\right)^{\frac{\alpha(n+1)}{2}} \sqrt{\alpha(1+n)+1}\left[\frac{\alpha(n+1)+1}{\alpha(n+1)}\right]^{\frac{\alpha(n+1)}{2}}
$$

and

$$
a_{0}(\alpha)=\frac{1}{\sqrt{\alpha(1+n)+1}} .
$$

Then $\varphi$ is increasing in $\left[0, a_{0}(\alpha)\right]$, decreasing in $\left[a_{0}(\alpha), 1\right]$ and $\varphi\left(a_{0}(\alpha)\right)=1$.

Theorem D. [2, Theorem 1.2] Suppose that $f \in H\left(\mathbf{B}^{n}, \mathbf{C}^{n}\right)$ such that $\|f\|_{\mathcal{P}(n, \alpha)}=$ 1 and $\operatorname{det} f^{\prime}(0)=\lambda \in(0,1]$. Then, for all $z$ with $|z| \leq \frac{a_{0}(\alpha)+m_{\alpha}(\lambda)}{1+a_{0}(\alpha) m_{\alpha}(\lambda)}$, we have

$$
\left|\operatorname{det} f^{\prime}(z)\right| \geq \operatorname{Re}\left(\operatorname{det} f^{\prime}(z)\right) \geq \frac{\lambda\left(m_{\alpha}(\lambda)-|z|\right)}{m_{\alpha}(\lambda)\left(1-m_{\alpha}(\lambda)|z|\right)^{\alpha(n+1)+1}},
$$


where $m_{\alpha}(\lambda)$ is the unique real root of the equation $\varphi(x)=\lambda$ in the interval $\left[0, a_{0}(\alpha)\right]$ and, $\varphi$ and $a_{0}(\alpha)$ are defined as in Lemma C. Moreover, for all $z$ with $|z| \leq \frac{a_{0}(\alpha)-m_{\alpha}(\lambda)}{1-a_{0}(\alpha) m_{\alpha}(\lambda)}$, we have

$$
\left|\operatorname{det} f^{\prime}(z)\right| \leq \frac{\lambda\left(m_{\alpha}(\lambda)+|z|\right)}{m_{\alpha}(\lambda)\left(1+m_{\alpha}(\lambda)|z|\right)^{\alpha(n+1)+1}} .
$$

Moreover, the estimates of (5) and (6) are sharp.

Proof of Theorem 1. Without loss of generality, we assume that $\|f\|_{\mathcal{P}(n, 1)}=1$ and $D_{f}^{n, 1}\left(z_{2}\right) \leq D_{f}^{n, 1}\left(z_{1}\right)$. Let $\phi \in \operatorname{Aut}\left(\mathbf{B}^{n}\right)$ such that $\phi(0)=z_{1}$ and $w=\phi^{-1}\left(z_{2}\right)$. For $z \in \mathbf{B}^{n}$, set $g=f(\phi(z))$. By [11, Proposition 1.21], we have

$$
\tanh \beta\left(z_{1}, z_{2}\right)=\tanh \beta\left(\phi^{-1}\left(z_{1}\right), \quad \phi^{-1}\left(z_{2}\right)\right)=\tanh \beta(0, w)=|w| .
$$

Since

we see that

$$
\left|\operatorname{det} \phi^{\prime}(z)\right|=\left(\frac{1-|\phi(z)|^{2}}{1-|z|^{2}}\right)^{\frac{n+1}{2}}
$$

$$
\left|\operatorname{det} g^{\prime}(0)\right|^{\frac{1}{n}}=\left|\operatorname{det} f^{\prime}(\phi(0))\right|^{\frac{1}{n}}\left|\operatorname{det} \phi^{\prime}(0)\right|^{\frac{1}{n}}=\left|\operatorname{det} f^{\prime}\left(z_{1}\right)\right|^{\frac{1}{n}}\left(1-\left|z_{1}\right|^{2}\right)^{\frac{n+1}{2 n}}
$$

and

$$
\begin{aligned}
\left(1-|w|^{2}\right)^{\frac{n+1}{2 n}}\left|\operatorname{det} g^{\prime}(w)\right|^{\frac{1}{n}} & =\left(1-|w|^{2}\right)^{\frac{n+1}{2 n}}\left|\operatorname{det} f^{\prime}(\phi(w))\right|^{\frac{1}{n}}\left|\operatorname{det} \phi^{\prime}(w)\right|^{\frac{1}{n}} \\
& =\left(1-|w|^{2}\right)^{\frac{n+1}{2 n}}\left|\operatorname{det} f^{\prime}\left(z_{2}\right)\right|^{\frac{1}{n}}\left(\frac{1-|\phi(w)|^{2}}{1-|w|^{2}}\right)^{\frac{n+1}{2 n}} \\
& =\left|\operatorname{det} f^{\prime}\left(z_{2}\right)\right|^{\frac{1}{n}}\left(1-\left|z_{2}\right|^{2}\right)^{\frac{n+1}{2 n}} .
\end{aligned}
$$

Case 1. If $\left|\operatorname{det} g^{\prime}(0)\right|=0$, then it is obvious.

Case 2. Let $\operatorname{det} g^{\prime}(0)=\lambda e^{i \theta}$, where $\lambda>0$ and $\theta \in[0,2 \pi]$. Applying Theorem D (5) to $e^{-i \frac{\theta}{n}} g(z)$, for $|z| \leq \frac{a_{0}(1)+m_{1}(\lambda)}{1+a_{0}(1) m_{1}(\lambda)}$, we have

$$
\operatorname{Re}\left(e^{-i \theta} \operatorname{det} g^{\prime}(z)\right) \geq \frac{\lambda\left(m_{1}(\lambda)-|z|\right)}{m_{1}(\lambda)\left(1-m_{1}(\lambda)|z|\right)^{n+2}},
$$

where $m_{1}(\lambda)$ and $a_{0}(1)$ are defined as in Theorem D.

Subcase 2.1. $|w| \leq m_{1}(\lambda)$. It is easy to know that

$$
m_{1}(\lambda) \leq \frac{a_{0}(1)+m_{1}(\lambda)}{1+a_{0}(1) m_{1}(\lambda)} .
$$

On the other hand, by calculations, we have

$$
\left(1-|w|^{2}\right)^{\frac{n+1}{2 n}} \geq\left(1-|w| m_{1}(\lambda)\right)^{\frac{n+1}{2 n}} \geq\left(1-|w| m_{1}(\lambda)\right)^{\frac{n+2}{n}}
$$

and

which gives

$$
m_{1}^{\frac{1}{n}}(\lambda)-|w|^{\frac{1}{n}} \leq\left(m_{1}(\lambda)-|w|\right)^{\frac{1}{n}}
$$

$$
m_{1}^{\frac{1}{n}}(\lambda)-\frac{\left(1-|w|^{2}\right)^{\frac{n+1}{2 n}}\left(m_{1}(\lambda)-|w|\right)^{\frac{1}{n}}}{\left(1-m_{1}(\lambda)|w|\right)^{\frac{n+2}{n}}} \leq|w|^{\frac{1}{n}} .
$$

By Lemma C and Theorem D, we have

$$
m_{1}(\lambda)\left(1-m_{1}^{2}(\lambda)\right)^{\frac{n+1}{2}}(M(n))^{n}=\lambda,
$$


which, together with (7), (8), (9), (10) and (11), implies that

$$
\begin{aligned}
D_{f}^{n, 1}\left(z_{1}\right)-D_{f}^{n, 1}\left(z_{2}\right) & =\left|\operatorname{det} g^{\prime}(0)\right|^{\frac{1}{n}}-\left(1-|w|^{2}\right)^{\frac{n+1}{2 n}}\left|\operatorname{det} g^{\prime}(w)\right|^{\frac{1}{n}} \\
& =\lambda^{\frac{1}{n}}-\left(1-|w|^{2}\right)^{\frac{n+1}{2 n}}\left|\operatorname{det} g^{\prime}(w)\right|^{\frac{1}{n}} \\
& \leq \lambda^{\frac{1}{n}}-\frac{\left(1-|w|^{2}\right)^{\frac{n+1}{2 n}} \lambda^{\frac{1}{n}}\left(m_{1}(\lambda)-|w|\right)^{\frac{1}{n}}}{m_{1}^{\frac{1}{n}}(\lambda)\left(1-m_{1}(\lambda)|w|\right)^{\frac{n+2}{n}}} \\
& =\left(\frac{\lambda}{m_{1}(\lambda)}\right)^{\frac{1}{n}}\left[m_{1}^{\frac{1}{n}}(\lambda)-\frac{\left(1-|w|^{2}\right)^{\frac{n+1}{2 n}}\left(m_{1}(\lambda)-|w|\right)^{\frac{1}{n}}}{\left(1-m_{1}(\lambda)|w|\right)^{\frac{n+2}{n}}}\right] \\
& \leq\left(\frac{\lambda}{m_{1}(\lambda)}\right)^{\frac{1}{n}}|w|^{\frac{1}{n}}=M(n)\left(1-m_{1}^{2}(\lambda)\right)^{\frac{1}{n}}|w|^{\frac{1}{n}} \leq M(n)|w|^{\frac{1}{n}} .
\end{aligned}
$$

Subcase 2.2. $|w|>m_{1}(\lambda)$. Then, by (7), (8) and (12)

$$
\begin{aligned}
D_{f}^{n, 1}\left(z_{1}\right)-D_{f}^{n, 1}\left(z_{2}\right) & =\left|\operatorname{det} g^{\prime}(0)\right|^{\frac{1}{n}}-\left(1-|w|^{2}\right)^{\frac{n+1}{2 n}}\left|\operatorname{det} g^{\prime}(w)\right|^{\frac{1}{n}} \\
& \leq \lambda^{\frac{1}{n}}=m_{1}^{\frac{1}{n}}(\lambda)\left(1-m_{1}^{2}(\lambda)\right)^{\frac{n+1}{2 n}} M(n)<M(n)|w|^{\frac{1}{n}} .
\end{aligned}
$$

Now we prove the sharpness part. For any $\epsilon \in(0, M(n)]$, let

$$
m_{1}^{*}(\lambda)=\min \left\{\left[1-\left(1-\frac{\epsilon}{M(n)}\right)^{\frac{2 n}{n+1}}\right]^{\frac{1}{2}}, a_{0}(1)\right\}
$$

and

$$
\lambda=m_{1}^{*}(\lambda)\left[1-\left(m_{1}^{*}(\lambda)\right)^{2}\right]^{\frac{n+1}{2}}(M(n))^{n} .
$$

By (13), we get

$$
M(n)\left[1-\left(m_{1}^{*}(\lambda)\right)^{2}\right]^{\frac{n+1}{2 n}} \geq M(n)-\epsilon .
$$

For $z \in \mathbf{B}^{n}$, let

$$
f_{\lambda}(z)=\left(\begin{array}{c}
\int_{0}^{z_{1}} \frac{\lambda\left(m_{1}^{*}(\lambda)-\xi\right)}{m_{1}^{*}(\lambda)\left(1-m_{1}^{*}(\lambda) \xi\right)^{n+2}} d \xi \\
z_{2} \\
\vdots \\
z_{n}
\end{array}\right),
$$

which gives that $\operatorname{det} f_{\lambda}^{\prime}(0)=\lambda$.

\section{Claim 1.}

$$
\left\|f_{\lambda}\right\|_{\mathcal{P}(n, 1)}=1 \text {. }
$$

Now we prove (16). For $z \in \mathbf{B}^{n}$, let

$$
F(z)=\left(\begin{array}{c}
-\frac{(M(n))^{n}}{2} z_{1}^{2} \\
z_{2} \\
\vdots \\
z_{n}
\end{array}\right)
$$

By Lemma C, we have

$$
\|F\|_{\mathcal{P}(n, 1)}=\sup _{z \in \mathbf{B}^{n}} D_{F}^{n, 1}(z)=1
$$


For $a=\left(m_{1}^{*}(\lambda), 0, \ldots, 0\right)$ and $z \in \mathbf{B}^{n}$, set

$$
\phi_{a}(z)=\left(\frac{m_{1}^{*}(\lambda)-z_{1}}{1-m_{1}^{*}(\lambda) z_{1}}, \frac{\left[1-\left(m_{1}^{*}(\lambda)\right)^{2}\right]^{\frac{1}{2}} z_{2}}{m_{1}^{*}(\lambda) z_{1}-1}, \ldots, \frac{\left[1-\left(m_{1}^{*}(\lambda)\right)^{2}\right]^{\frac{1}{2}} z_{n}}{m_{1}^{*}(\lambda) z_{1}-1}\right) \in \operatorname{Aut}\left(\mathbf{B}^{n}\right) .
$$

Then, by (14), we have

$$
\begin{aligned}
\left|\operatorname{det} f_{\lambda}^{\prime}(z)\right| & =\frac{(M(n))^{n}\left|m_{1}^{*}(\lambda)-z_{1}\right|\left[1-\left(m_{1}^{*}(\lambda)\right)^{2}\right]^{\frac{n+1}{2}}}{\left|1-m_{1}^{*}(\lambda) z_{1}\right|^{2+n}} \\
& =\left|\operatorname{det}\left(F\left(\phi_{a}(z)\right)\right)^{\prime}\right| \\
& =\left|\operatorname{det} F^{\prime}\left(\phi_{a}(z)\right)\right|\left|\operatorname{det} \phi_{a}^{\prime}(z)\right| \\
& =\frac{\left|\operatorname{det} F^{\prime}\left(\phi_{a}(z)\right)\right|\left(1-\left|\phi_{a}(z)\right|^{2}\right)^{\frac{n+1}{2}}}{\left(1-|z|^{2}\right)^{\frac{n+1}{2}}},
\end{aligned}
$$

which, together with (17), implies that

$$
\begin{aligned}
D_{f_{\lambda}}^{n, 1}(z) & =\left(1-|z|^{2}\right)^{\frac{n+1}{2 n}}\left|\operatorname{det}\left(F\left(\phi_{a}(z)\right)\right)^{\prime}\right|^{\frac{1}{n}} \\
& =\left|\operatorname{det} F^{\prime}\left(\phi_{a}(z)\right)\right|^{\frac{1}{n}}\left(1-\left|\phi_{a}(z)\right|^{2}\right)^{\frac{n+1}{2 n}}=D_{F}^{n, 1}\left(\phi_{a}(z)\right) \leq 1 .
\end{aligned}
$$

Hence (16) follows from (18). The proof of Claim 1 is finished.

Therefore, for $w^{\prime}=(0,0, \ldots, 0)$ and $w^{\prime \prime}=\left(m_{1}^{*}(\lambda), 0, \ldots, 0\right)$, we have

$$
\tanh \beta\left(w^{\prime}, w^{\prime \prime}\right)=m_{1}^{*}(\lambda)
$$

which, together with (14), (15) and (16), yields that

$$
\begin{aligned}
\left|D_{f_{\lambda}}^{n, 1}\left(w^{\prime}\right)-D_{f_{\lambda}}^{n, 1}\left(w^{\prime \prime}\right)\right| & =\lambda^{\frac{1}{n}}=M(n)\left(m_{1}^{*}(\lambda)\right)^{\frac{1}{n}}\left[1-\left(m_{1}^{*}(\lambda)\right)^{2}\right]^{\frac{n+1}{2 n}} \\
& \geq\left\|f_{\lambda}\right\|_{\mathcal{P}(n, 1)}\left[\tanh \beta\left(w^{\prime}, w^{\prime \prime}\right)\right]^{\frac{1}{n}}(M(n)-\epsilon) .
\end{aligned}
$$

The above inequality shows that the constant $M(n)$ is sharp. The proof of this theorem is complete.

Proof of Theorem 2. For $z=x+i y \in \mathbf{D}$, let $w=z+r e^{i \theta}$. Then, by Theorem 1, we have

$$
\begin{aligned}
\Lambda_{f}(z) & =\max _{\theta \in[0,2 \pi]}\left[\lim _{r \rightarrow 0^{+}}\left(\frac{\left|D_{f}^{1,1}(z)-D_{f}^{1,1}(w)\right|}{r} \cdot \frac{r}{\rho(z, w)}\right)\right] \\
& =\left(1-|z|^{2}\right) \max _{\theta \in[0,2 \pi]}\left|\frac{\partial}{\partial x} D_{f}^{1,1}(z) \cos \theta+\frac{\partial}{\partial y} D_{f}^{1,1}(z) \sin \theta\right| \\
& =\frac{\left(1-|z|^{2}\right)}{2}\left(\left|\frac{\partial}{\partial x} D_{f}^{1,1}(z)+i \frac{\partial}{\partial y} D_{f}^{1,1}(z)\right|+\left|\frac{\partial}{\partial x} D_{f}^{1,1}(z)-i \frac{\partial}{\partial y} D_{f}^{1,1}(z)\right|\right) \\
& =\left(1-|z|^{2}\right)\left(\left|\frac{\partial}{\partial z} D_{f}^{1,1}(z)\right|+\left|\frac{\partial}{\partial \bar{z}} D_{f}^{1,1}(z)\right|\right) \leq \frac{3 \sqrt{3}}{2}\|f\|_{\mathcal{P}(1,1)},
\end{aligned}
$$

where

$$
\Lambda_{f}(z)=\max _{\theta \in[0,2 \pi]}\left(\lim _{r \rightarrow 0^{+}} \frac{\left|D_{f}^{1,1}(z)-D_{f}^{1,1}(w)\right|}{\rho(z, w)}\right) .
$$


On the other hand,

$$
\begin{aligned}
\left|\frac{\partial}{\partial z} D_{f}^{1,1}(z)\right|+\left|\frac{\partial}{\partial \bar{z}} D_{f}^{1,1}(z)\right|= & |-\bar{z}| f^{\prime}(z)\left|+\frac{f^{\prime \prime}(z) \overline{f^{\prime}(z)}}{2\left|f^{\prime}(z)\right|}\left(1-|z|^{2}\right)\right| \\
& +|-z| f^{\prime}(z)\left|+\frac{\overline{f^{\prime \prime}(z)} f^{\prime}(z)}{2\left|f^{\prime}(z)\right|}\left(1-|z|^{2}\right)\right| \\
\geq & \left|f^{\prime \prime}(z)\right|\left(1-|z|^{2}\right)-2|z|\left|f^{\prime}(z)\right|,
\end{aligned}
$$

which, together with (19), gives that

$$
\begin{aligned}
\left|f^{\prime \prime}(z)\right|\left(1-|z|^{2}\right) & \leq 2|z|\left|f^{\prime}(z)\right|+\left|\frac{\partial}{\partial z} D_{f}^{1,1}(z)\right|+\left|\frac{\partial}{\partial \bar{z}} D_{f}^{1,1}(z)\right| \\
& \leq \frac{2|z|\|f\|_{\mathcal{P}(1,1)}}{1-|z|^{2}}+\frac{\frac{3 \sqrt{3}}{2}\|f\|_{\mathcal{P}(1,1)}}{1-|z|^{2}}=\frac{\left(2|z|+\frac{3 \sqrt{3}}{2}\right)\|f\|_{\mathcal{P}(1,1)}}{\left(1-|z|^{2}\right)} .
\end{aligned}
$$

The proof of this theorem is complete.

Proof of Theorem 3. Without loss of generality, we assume that $\|f\|_{\mathcal{P}(n, 1)}=1$. For $z \in \mathbf{B}^{n}$, it follows from Theorem 1 that there is a point $w \in \mathbf{B}^{n}$ such that

$$
D_{f}^{n, 1}(w)>1-\sigma
$$

and

$$
\left|D_{f}^{n, 1}(w)-D_{f}^{n, 1}(z)\right| \leq\left[M(n)\left(\frac{n+1}{n+2}\right)^{\frac{1}{n}}+\sigma\right][\tanh \beta(w, z)]^{\frac{1}{n}}
$$

where

$$
\sigma=\frac{1-r M(n)\left(\frac{n+1}{n+2}\right)^{\frac{1}{n}}}{2(1+r)} \text { and } \quad r<\frac{1}{M(n)}\left(\frac{n+2}{1+n}\right)^{\frac{1}{n}} .
$$

By the assumption, there is a point $z_{w}$ such that

$$
\left[\tanh \beta\left(\varphi\left(z_{w}\right), w\right)\right]^{\frac{1}{n}}<r<\frac{1}{M(n)}\left(\frac{n+2}{1+n}\right)^{\frac{1}{n}}
$$

and $\left|\tau_{\varphi}\left(z_{w}\right)\right|>\varepsilon$, which imply that

$$
\begin{aligned}
D_{f_{\lambda}}^{n, 1}\left(\varphi\left(z_{w}\right)\right) & \geq D_{f_{\lambda}}^{n, 1}(w)-\left[M(n)\left(\frac{n+1}{n+2}\right)^{\frac{1}{n}}+\sigma\right]\left[\tanh \beta\left(\varphi\left(z_{w}\right), w\right)\right]^{\frac{1}{n}} \\
& \geq 1-\sigma-\left[M(n)\left(\frac{n+1}{n+2}\right)^{\frac{1}{n}}+\sigma\right] r \\
& =1-r M(n)\left(\frac{n+1}{n+2}\right)^{\frac{1}{n}}-(1+r) \sigma \\
& =\frac{1-r M(n)\left(\frac{n+1}{n+2}\right)^{\frac{1}{n}}}{2}>0
\end{aligned}
$$

By (19), we conclude that

$$
\left\|C_{\varphi}(f)\right\|_{\mathcal{P}(n, 1)} \geq D_{f_{\lambda}}^{n, 1}\left(\varphi\left(z_{w}\right)\right)\left|\tau_{\varphi}\left(z_{w}\right)\right|>k(n, r, \varepsilon),
$$


where

$$
k(n, r, \varepsilon)=\frac{\left[1-r M(n)\left(\frac{n+1}{n+2}\right)^{\frac{1}{n}}\right] \varepsilon}{2} .
$$

The proof of this theorem is compete.

Acknowledgement. This research of the first author was partly supported by the National Natural Science Foundation of China (No. 11401184 and No. 11571216), the Construct Program of the Key Discipline in Hunan Province, the Science and Technology Plan of Hunan Province (2016TP1020), the Fifty-ninth Batch of Post Doctoral Foundation of China (No. 2016M590492) and the Post Doctoral Foundation of Jiangsu Province (No. 1601182C).

\section{References}

[1] Chen, H. H., and P. Gauthier: Boundedness from below of composition operators on $\alpha$ Bloch spaces. - Canad. Math. Bull. 52, 2008, 195-204.

[2] Chen, S. L., S. Ponnusamy, and X. T. Wang Landau-Bloch constants for functions in $\alpha$ Bloch spaces and Hardy spaces. - Complex Anal. Oper. Theory 6, 2012, 1025-1036.

[3] Ghatage, P., J. Yan, and D. Zheng: Composition operators with closed range on the Bloch space. - Proc. Amer. Math. Soc. 129, 2000, 2039-2044.

[4] Hosokawa, T., and S. Ohno: Differences of weighted composition operators acting from Bloch space to $H^{\infty}$. - Trans. Amer. Math. Soc. 363, 2011, 5321-5340.

[5] Hosokawa, T., and S. Ohno: Differences of composition operators on the Bloch spaces. - J. Operat. Theor. 57, 2007, 229-242.

[6] LiU, X. Y.: Bloch functions of several complex variables. - Pacific J. Math. 152, 1992, 347-363.

[7] Madigan, K., and A. Matheson: Compact composition operators on the Bloch space. Trans. Amer. Math. Soc. 347, 1995, 2679-2687.

[8] Rudin, W.: Function theory in the unit ball of $\mathbf{C}^{n}$. - Spring-Verlag, New York, Heidelberg, Berlin, 1980.

[9] Xiong, C. J.: On the Lipschitz continuity of the dilation of Bloch functions. - Period. Math. Hung. 47, 2003, 233-238.

[10] ZHU, K.: Bloch type spaces of analytic functions. - Rocky Mountain J. Math. 23, 1993, 11431177.

[11] ZHU, K.: Spaces of holomorphic functions in the unit ball. - Springer, New York, 2005. 